\section{The Buteyko Technique: News}

\author{
Patrick McKeown, MA, BBE
}

Buteyko Clinic International

Comments by Dr. Masse in the editorial titled "The Buteyko Technique: Fake News or No News?"1 , overlook an increasing body of evidence supporting the therapeutic role of breathing re-education in the management of obstructive sleep apnea.

The basic tenets of breathing re-education include:

- Determining chemosensitivity to carbon dioxide using breath hold time

- Restoring nasal breathing on a permanent basis

- Adopting correct tongue resting posture

- Slowing the respiratory rate towards normal

- Restoring diaphragmatic function

- Achieving normal minute ventilation

Traditionally, obstructive sleep apnea was primarily regarded as an anatomical issue quantified by pharyngeal critical closing pressure (Pcrit). Recently, three non-anatomical traits have been recognised as contributory factors. ${ }^{2}$ These include loop gain, arousal threshold and upper airway recruitment. Eckert et al. reported that $69 \%$ of OSA patients have one or more predisposing physiological traits. $^{3}$

Oral breathing during sleep is six times more likely to occur in individuals over 40 years of age, ${ }^{4}$ affects resting tongue posture as the tongue falls into the airway, ${ }^{5}$ causes upper chest breathing and reduces pharyngeal airway space. Conversely, nasal breathing provides greater recruitment of the diaphragm, ${ }^{6}$ and increases lung volume to cause stiffening of the tracheal wall to help prevent collapse of the upper airways. ${ }^{7}$

Aside from anatomical compromise, 'elevated' loop gain is arguably the strongest determinant of OSA, with approximately one third of OSA patients having high loop gain. ${ }^{8} \mathrm{~A}$ high loop gain helps to predict favourable outcomes to non-anatomical interventions and an unfavourable outcome to anatomical interventions such as oral appliances or surgery. ${ }^{8}$ Individuals with high loop gain have an exaggerated response to minimal changes of carbon dioxide and experience excessive ventilation upon resumption of breathing. This causes hypocapnia, reducing the neural drive to breathe and thus, contributing to perpetuating apneas. $^{7}$
High loop gain during sleep can be estimated by simply measuring the length of time that subjects can voluntarily hold their breath. ${ }^{8}$ This forms part of breathing reeducation and provides useful feedback of the chemosensitivity to carbon dioxide. Furthermore, breathing re-education involving slow breathing depresses the hypercapnic response to reduce loop gain. ${ }^{9}$

The third phenotype is arousal threshold, which is the propensity to wake from sleep. Breathing through the nose during sleep improves arousal threshold, as sleep becomes deeper. ${ }^{10}$ Aside from sedatives, slow breathing through the nose improves arousal threshold. ${ }^{11}$

The final phenotype, upper airway recruitment, is the magnitude of stimuli required to recruit upper airway dilator muscles. Approximately $30 \%$ of persons with OSA have poor muscle responsiveness to airway narrowing during sleep. ${ }^{2}$ Nasal breathing harnesses the gas nitric oxide, which plays a role in the maintenance of muscle tone and regulation of neuromuscular pathways in the pharyngeal muscles. $^{5}$

In summary, individuals with obstructive sleep apnea commonly breathe orally, resulting in fast, shallow breathing using the upper chest. Abnormal breathing during sleep will affect all four phenotypes of sleep apnea. Breathing reeducation to restore nasal, slow and diaphragmatic breathing offers a therapeutic intervention to all patients with sleep apnea, regardless of phenotype.

\section{CITATION}

McKeown, P. The Buteyko technique: News. J Dent Sleep Med. 2019;6(2)

\section{REFERENCES}

1. Masse JF. The Buteyko technique: fake news or no news. Journal of Dental Sleep Medicine. 2018;5(3):53-54.

2. Osman AM, Carter SG, Carberry JC, Eckert DJ. Obstructive sleep apnea: current perspectives. Nat Sci Sleep. 2018;10:21-34. doi:10.2147/NSS.S124657

3. Eckert DJ, White DP, Jordan AS, Malhotra A, Wellman A. Defining phenotypic causes of obstructive sleep apnea: Identification of novel therapeutic targets. Am J Respir Crit Care Med. 2013;188(8):9961004. doi:10.1164/rccm.201303-0448OC

4. Madronio M, Di Somma E, Stavrinou R et al. Older individuals have increased oro-nasal breathing during sleep. European Respiratory 
Journal. 2004;24(1):71-77. doi:10.1183/09031936.04.00004303

5.

Michels D de S, Rodrigues A da MS, Nakanishi M, Sampaio ALL, Venosa AR. Nasal Involvement in Obstructive Sleep Apnea Syndrome. Int J Otolaryngol. 2014;2014:1-8. doi:10.1155/2014/717419

6.

Trevisan ME, Boufleur J, Soares JC, Haygert CJP, Ries LGK, Corrêa ECR. Diaphragmatic amplitude and accessory inspiratory muscle activity in nasal and mouth-breathing adults: A cross-sectional study. $J$ Electromyogr Kinesiol. 2015;25(3):463-468. doi:10.1016/j.jelekin.2015.03.006

7. Deacon NL, Jen R, Li Y, Malhotra A. Treatment of obstructive sleep apnea prospects for personalized combined modality therapy. Ann Am Thorac Soc. 2016;13(1):101-108. doi:10.1513/AnnalsATS.201508537FR

8. Messineo L, Taranto-Montemurro L, Azarbarzin A, et al. Breathholding as a means to estimate the loop gain contribution to obstructive sleep apnoea. $J$ Physiol. 2018;596(17):4043-4056. doi:10.1113/JP276206

9. Bernardi L, Gabutti A, Porta C, Spicuzza L. Slow breathing reduces chemoreflex response to hypoxia and hypercapnia, and increases baroreflex sensitivity. J Hypertens. 2001;19(12):2221-2229. http://www.ncbi.nlm.nih.gov/pubmed/11725167. Accessed April 2, 2019.

10. Zwillich CW, Pickett C, Hanson FN, Weil J V. Disturbed sleep and prolonged apnea during nasal obstruction in normal men. Am Rev
Respir Dis. 1981;124(2):158-160. doi:10.1164/arrd.1981.124.2.158.

11. Goldman B. Study shows how slow breathing induces tranquility. Stanford Medicine News Center. https://med.stanford.edu/news/allnews/2017/03/study-discovers-how-slow-breathing-induces-tranquility.html. Accessed March 26, 2019.

\section{SUBMISSION AND CORRESPONDENCE INFORMATION}

Submitted in final revised form March 27, 2019
Accepted for publication March 31, 2019

Address correspondence to: Patrick McKeown. MA, BBE, Buteyko Clinic International; Email: patrick@buteykoclinic.com

\section{DISCLOSURE STATEMENT}

The author is a Buteyko Breathing Educator. 